\title{
Enhanced Emotional Empathy after Mineralocorticoid Receptor Stimulation in Women with Borderline Personality Disorder and Healthy Women
}

\author{
Katja Wingenfeld*,', Linn K Kuehl', Katrin Janke', Kim Hinkelmann', Isabel Dziobek², Juliane Fleischer', \\ Christian Otte ${ }^{1,3}$ and Stefan Roepke ${ }^{1,3}$
}

'Department of Psychiatry and Psychotherapy, Charité University Medical School Berlin, Campus Benjamin Franklin, Berlin, Germany; ${ }^{2}$ Cluster of Excellence Languages of Emotion, Freie Universität Berlin, Berlin, Germany

\begin{abstract}
The mineralocorticoid receptor (MR) is highly expressed in the hippocampus and prefrontal cortex. MR have an important role in appraisal processes and in modulating stress-associated emotional reactions but it is not known whether the MR affects empathy. Borderline personality disorder (BPD) is characterized by disturbed emotion regulation and alterations in empathy. In the current study, we examined whether stimulation of the MR enhances empathy in patients with BPD and healthy individuals. In a placebo-controlled study, we randomized 38 women with BPD and without psychotropic medication, and 35 healthy women to either placebo or $0.4 \mathrm{mg}$ fludrocortisone, an MR agonist. Subsequently, all participants underwent two tests of social cognition, the Multifaceted Empathy Test (MET) and the Movie for the Assessment of Social Cognition (MASC), measuring cognitive and emotional facets of empathy. Eighteen BPD patients and 18 healthy women received placebo, whereas 20 BPD patients and 17 healthy women received fludrocortisone. In the MET, fludrocortisone enhanced emotional empathy across groups, whereas cognitive empathy was not affected. In the MASC, no effect of fludrocortisone could be revealed. In both tests, BPD patients and healthy women did not differ significantly in cognitive and emotional empathy and in their response to fludrocortisone. Stimulation of MR enhanced emotional empathy in healthy women and in BPD patients. Whether fludrocortisone might have a therapeutic role in psychotherapeutic processes, remains to be elucidated.

Neuropsychopharmacology (2014) 39, 1799-1804; doi: I0.1038/npp.2014.36; published online I2 March 2014
\end{abstract}

\section{INTRODUCTION}

Stress leads to an increase in glucocorticoid secretion, ie, cortisol in humans and cortisone in rodents, which influence a wide range of cognitive and emotional functions such as memory performance, fear-motivated behavior, or stress-associated emotional reactions (de Kloet, 2013). Glucocorticoids mediate their effects by binding to two receptors, the mineralocorticoid receptor (MR) and the glucocorticoid receptor (GR), which differ in their affinity and distribution within the brain (Lupien and Lepage, 2001; de Kloet et al, 2005; Joels et al, 2008; Roozendaal et al, 2010). Although most of the effects of GCs have been attributed to GR, more recent studies emphasize the importance of MR (Joels et al, 2008; de Kloet, 2010; Harris et al, 2012). Indeed, it has been consistently shown that blocking the MR leads to impaired cognitive function

\footnotetext{
*Correspondence: Dr K Wingenfeld, Department of Psychiatry and Psychotherapy, Charité University Medical School Berlin, Campus Benjamin Franklin, Eschenallee 3, Berlin 14050, Germany. Tel: +49 308445 8708, Fax: +49 3084458255 ,

E-mail: katja.wingenfeld@charite.de

${ }^{3}$ Christian Otte and Stefan Roepke share the last authorship.

Received 9 December 2013; revised 10 January 2014; accepted 12

February 20।4; accepted article preview online 18 February 2014
}

in humans (Otte et al, 2007; Cornelisse et al, 2011; Rimmele et al, 2013). Interestingly, these impairing effects of MR blockade were most pronounced for emotional memory (Rimmele et al, 2013). This fits very well with animal data showing that MR are particularly involved in the appraisal of novel situations and in modulating stress-associated emotional reactions (Ter Horst et al, 2012; de Kloet, 2013; Kruk et al, 2013).

As humans often have to perform complex social cognitive tasks while being stressed, it is of great interest to understand how stress and stress hormones influence social cognition, ie, the ability to process, store, and use information about other people and social situations. A phenomenon closely related to social cognition is empathy, which consists of at least two components: the first is a cognitive component that captures the capacity to infer others' mental states and is also referred to as perspective taking, mentalizing, or theory of mind (Zaki and Ochsner, 2012). Second, empathy also comprises an affective component, ie, an emotional response to another person's emotional state (Blair, 2008; Roepke et al, 2012). One task to investigate both components of empathy is the 'Multifaceted Empathy Test' (MET) (Dziobek et al, 2008). Another task that was developed to assess cognitive empathy in a more ecologically valid fashion, is the 'Movie for the 
Assessment of Social Cognition' (MASC) (Dziobek et al, 2006), which is a video-based task demanding test takers to infer emotions, thoughts, and intentions of characters engaged in social interactions. An elegant study used the MASC to investigate the association between the cortisol response to a psychosocial stressor and cognitive empathy (Smeets et al, 2009). In women, elevated cognitive empathy was found for those who responded with low cortisol to stress exposure. Furthermore, in women, there was a negative association between cortisol reactivity and MASC scores (Smeets et al, 2009). In men, an opposite pattern was seen, with high cortisol responders showing greater MASC scores and a negative correlation between stress hormones and empathy. Thus, this study suggests sex-specific effects of glucocorticoids on social cognition.

Deficits in social cognition are discussed for several mental disorders, including borderline personality disorder (BPD) (Roepke et al, 2012). Of note, many of the symptoms seen in BPD occur within social contexts, leading to the assumption that BPD might be characterized by aberrant social cognition. Indeed, impairments were seen in previous studies using the MET to assess cognitive and emotional empathy (Preissler et al, 2010; Dziobek et al, 2011). In the emotional empathy items of the MET, participants were required to rate the amount of mirroring of an emotion that took place in response to a picture (eg, if the mental state of the person was anxious, subjects were asked to rate how anxious they felt) and additionally rated the degree of empathic concern they felt for the person in the picture. Results from the MET revealed that BPD patients had significantly reduced tendencies to feel empathy for other people in emotionally distressing situations compared with non-clinical controls (Dziobek et al, 2011).

On the basis of the findings of a negative association between cortisol reactivity and cognitive empathy in women (Smeets et al, 2009) and the impairing effects of selective MR blockade on emotional memory and cognitive performance (Otte et al, 2007; Cornelisse et al, 2011; Rimmele et al, 2013), we hypothesized that selective MR stimulation with fludrocortisone would enhance cognitive and emotional empathy in healthy women. Furthermore, we included a clinical group of patients with BPD for which deficits in cognitive and emotional empathy have been demonstrated (Dziobek et al, 2011). We hypothesized that fludrocortisone would improve cognitive and emotional empathy in BPD.

\section{MATERIALS AND METHODS}

\section{Participants}

In total, 38 women with BPD and 35 healthy women were recruited and completed the study. All participants were free of psychotropic medication. Participants were excluded if they had any of the following medical conditions: CNS diseases or severe somatic diseases, metabolic or endocrine diseases, autoimmune diseases, current infections, or pregnancy. Further exclusion criteria were schizophrenia, schizoaffective disorder, bipolar disorder, depressive disorder with psychotic features, anorexia, alcohol or drug abuse, and dependence in the last 6 months (all assessed by MINI-International Neuropsychiatric Interview). All patients had negative urine drug screening (benzodiazepines, opiates, cocaine, amphetamines, and cannabinoids) during hospital admission. Written informed consent was obtained from all participants. Healthy participants were recruited by local advertisement and received financial remuneration (80€). The study was approved by the Medical Councils' Ethics Committee of Hamburg and Berlin.

\section{Procedure}

We used the MINI to assess current psychiatric diagnoses (Sheehan et al, 1998, German version Ackenheil et al, 1999). BPD was diagnosed using the Structured Clinical Interview for DSM-IV axis II (First et al, 1997). Clinical interviews were conducted by two trained $\mathrm{PhD}$ psychologists (KJ and LK).

A placebo-controlled study was performed and participants were randomized (simple randomization) to either $0.4 \mathrm{mg}$ fludrocortisone (Astonin $\mathrm{H}$, MerckSerono) orally or to placebo. Drugs were administered at 14:00 $\mathrm{h}$ and testing took place between 15:45 and 16:45 h. Fludrocortisone exhibits its maximum effects after $1.7 \mathrm{~h}$ after drug intake (DRUGDEX). We did not use a within-subject design because no parallel versions of the MET and MASC were available. The participants were tested in a quiet room and were allowed to drink some water.

\section{Social Cognition Tasks}

MET. To assess cognitive and emotional empathy, the MET was used (Dziobek et al, 2008) in a modified version (Hurlemann et al, 2007; Dziobek et al, 2011; Ritter et al, 2011). The MET is a PC-assisted test consisting of photographs that show 30 picture stimuli with people in emotionally charged situations. To assess cognitive empathy, participants were required to infer the mental state of the subject in the photo and were asked to indicate the correct one from a list of four. To assess emotional empathy, participants were asked to rate the degree of empathic concern they felt for the person in the picture (Likert scale, $0=$ not at all, $9=$ very much). Pictures were presented in six blocks of 10 picture stimuli. In the first block, participants were asked for cognitive empathy. In the next blocks, it was asked for emotional and cognitive empathy in alternating order. In this way, each picture was rated for cognitive and emotional empathy. The score range is $0-30$ for the cognitive empathy and $30-270$ for the emotional empathy.

MASC. In addition, we administered the MASC, a sensitive video-based test for the evaluation of cognitive empathy (Dziobek et al, 2006; Preissler et al, 2010; Ritter et al, 2011). This task involves watching a $15 \mathrm{~min}$ movie about four characters spending an evening together and answering questions referring to the actors' mental states. The movie stops 45 times, when questions about the actors' feelings/emotions (score range $0-15$ ), thoughts (score range $0-4$ ), and intentions (score range 0-14) are asked. Participants are required to choose the correct answer out of four possible ones. 


\section{Statistical Analysis}

Statistical analyses were performed using SPSS Version 18.0. Demographic data were analyzed using Pearson's $\chi^{2}$-test for categorical data and Student's $t$-test for continuous data. Effects of fludrocortisone on social cognition were analyzed using analysis of variance (ANOVA) with the main factors treatment (fludrocortisone vs placebo) and group (BPD vs controls). In a second step, all analyses were controlled for BMI, years of school education (covariate), smoking, and intake of oral contraceptives (additional factor).

\section{RESULTS}

\section{Demographic and Clinical Data}

In BPD patients, the following current comorbid axis I disorders were reported: major depressive disorder $n=9$, dysthymia $n=5$, panic disorder $n=2$, agoraphobia $n=5$, social phobia $n=7$, obsessive compulsive disorder $n=2$, PTSD $n=5$, bulimia nervosa $n=2$, substance abuse $n=5$, and alcohol abuse $n=2$. None of the patients fulfilled the criteria of antisocial personality disorder.

BPD patients and healthy women did not differ with regard to age. Healthy women had slightly more years of education and had a slightly lower body mass index. There were more smokers in the patient group, whereas more healthy women took oral contraceptives (sample characteristics are presented in Table 1). All variables that differed significantly between patients and healthy women were controlled for in statistical analyses.

\section{Effects of Fludrocortisone vs Placebo on Cognitive and Emotional Empathy}

In our placebo-controlled study, $18 \mathrm{BPD}$ patients and 18 healthy women received placebo, whereas 20 BPD patients and 17 healthy women received fludrocortisone. There were missing data for two BPD patients in the MASC.

\section{MET}

First, we performed an ANOVA with repeated measurements analyzing the effect of fludrocortisone (betweensubject factor 'treatment') on emotional and cognitive empathy (within-subject factor 'test score') in patients with BPD and healthy control (between-subject factor 'group'). A main effect treatment $(p=0.008)$ as well as a test score by treatment-interaction effect $(p=0.01)$ could be revealed, whereas there was no main effect group $(p=0.13)$ or treatment by group interaction effect $(p=0.56)$. This result suggests a differential effect on fludrocortisone on emotional and cognitive empathy.

To clarify this test score by treatment-interaction effect, separate ANOVAs were conducted to further analyze the effects of fludrocortisone on cognitive and emotional empathy with group (BPD $v s$ controls) and treatment (fludrocortisone $v s$ placebo) as between-subject factors.

Emotional empathy was enhanced in the fludrocortisone condition compared with placebo across groups (main effect treatment $\left.F_{\mathrm{df} 1,69}=7,1, p=0.009\right)$. There was no significant group $\left(F_{\mathrm{df} 1,69}=2,1, p=0.15\right)$ or treatment by group interaction $\left(F_{\mathrm{df1} 169}=0,4, p=0.55\right)$ effect, suggesting a similar effect of fludrocortisone on emotional empathy across groups (see Figure 1) and no differences between BPD patients and healthy women in emotional empathy across treatment modalities (BPD mean: 148 (49) range 30239; controls mean: 161 (42) range 61-266). The results did not change in the ANCOVA controlling for the abovementioned confounders, ie, body mass index, smoking, and education. The improving effect of fludrocortisone on emotional empathy was seen for positive $(p=0.016)$ as well as for negative $(p=0.041)$ emotions. The effect size (Cohen's $d$ ) of the effect of fludrocortisone on emotional empathy was $d=0.63$ across groups, indicating a medium effect (BPD patients $d=0.73$, healthy controls $d=0.53$ ).

In the cognitive part of the MET, there was no effect of the main factor treatment and no significant group effect or treatment by group interaction (all $p$-values $>0.55$ ) could be revealed. Thus, there was no difference between BPD patients and healthy women in cognitive empathy across treatment modalities (BPD mean (SD): 19.5 (2.9) range 1426; controls mean: 20.3 (3.1) range 14-27). Controlling for potentially confounding variables using ANCOVA did not change these results.

\section{MASC}

Separate ANOVAs were conducted to analyze the effects of fludrocortisone on cognitive empathy, ie, the inferring of emotions, thoughts, and intentions in the MASC with group (BPD vs controls) and treatment (fludrocortisone $v s$ placebo) as between-subject factors. Mean values and SD are presented in Table 2.

Concerning the subscore 'emotions', we found a significant effect of the main factor group in unadjusted analyses $\left(F_{\mathrm{df} 1,67}=4,31, p=0.04\right)$ but this effect did not hold after controlling for potential confounders $(p=0.68)$. There was no main effect of treatment or group by treatmentinteraction effect (both $p$-values $>0.28$ ).

Table I Sample Characteristics

\begin{tabular}{lccc}
\hline & BPD & Healthy women & Statistics \\
\hline Age (mean (SD)) & $24.3(5.7)$ & $25.5(5.6)$ & $t_{\mathrm{df} / \mid}=-0.86, p=0.40$ \\
Years of school education (mean (SD)) & $10.2(1.5)$ & $11.0(1.5)$ & $t_{\mathrm{df} 67}=-2.3 \mid, p=0.02$ \\
Body mass index (mean (SD)) & $26.0(7.1)$ & $23.6(5.0)$ & $t_{\mathrm{df} \mid}=-1.65, p=0.10$ \\
Smoker (yes/no) & $27 / 1 \mid$ & $10 / 24$ & $\chi^{2}=|2.45, p<0.00|$ \\
Intake of oral contraceptives (yes/no) & $9 / 29$ & $16 / 19$ & $\chi^{2}=4.76, p=0.03$ \\
\hline
\end{tabular}

Abbreviation: BPD, borderline personality disorder. 

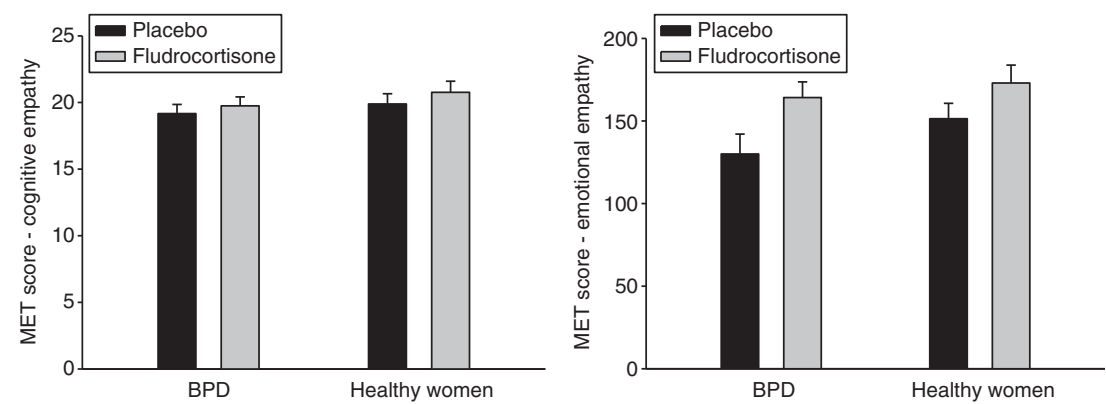

Figure I Cognitive and emotional empathy measured with the Multifaceted Empathy Test (MET) after fludrocortisone administration and placebo in patients with BPD and healthy women. A significant main effect of treatment was found for the emotional test part $(p=0.009)$.

Table 2 Cognitive Empathy Measured with the MASC after Fludrocortisone Administration and Placebo in Patients with BPD and Healthy Women

\begin{tabular}{lcc}
\hline MASC (mean/SD) & BPD & Healthy women \\
\hline Placebo & $n=17$ & $n=18$ \\
Recognition of emotions & $|1.4|(2.2)$ & $12.06(1.4)$ \\
Recognition of thoughts & $3.29(0.7)$ & $3.28(0.8)$ \\
Recognition of intentions & $10.94(2.1)$ & $10.33(0.9)$ \\
Fludrocortisone & $n=19$ & $n=17$ \\
Recognition of emotions & $10.53(2.0)$ & $11.88(2.4)$ \\
Recognition of thoughts & $3.16(1.0)$ & $3.12(1.0)$ \\
Recognition of intentions & $9.79(1.9)$ & $10.59(2.7)$ \\
\hline
\end{tabular}

Abbreviations: BPD, borderline personality disorder; MASC, Movie for the Assessment of Social Cognition.

No significant main effect of group, treatment, or group by treatment-interaction effect (all $p$-values $>0.49$ ) could be revealed for the subscore 'thought'. Similarly, there was no significant main effect of group, treatment, or group by treatment-interaction effect (all $p$-values $>0.15$ ) for the subscore 'intentions'. These results did not change after controlling for potentially confounding variables.

\section{DISCUSSION}

This study examined the effects of MR stimulation via fludrocortisone administration on cognitive and emotional empathy in women with BPD and in healthy women. In line with our hypothesis, MR stimulation led to enhanced emotional empathy, whereas, contrary to our hypothesis, cognitive empathy was not clearly influenced by fludrocortisone. Remarkably, we found enhanced emotional empathy after fludrocortisone across groups, in healthy women as well as in women with BPD. Patients and healthy participants did not differ significantly in cognitive and emotional empathy across treatment modalities.

This is the first study to examine the effects of MR stimulation on social cognition in humans. Our result that fludrocortisone enhances emotional empathy fits very well with animal data. MR are particularly involved in the appraisal of novel situations and in selection of response strategies (de Kloet, 2013). In this context, MR are important in modulating stress-associated emotional arousal and adaptive behaviors (Brinks et al, 2007). Of note, in a stressful situation, the individual needs to decide immediately how to act and emotional appraisal and reaction is often essential for fast reaction in the context of perceived danger and anxiety. MR are expressed in high density in limbic brain areas, which are involved in the processing of emotional information (Joels et al, 2011; Groeneweg et al, 2012). Indeed, blockade of MR impaired memory performance particularly for emotional material (Rimmele et al, 2013). Therefore, it is plausible that MR influences emotional empathy given that emotional empathy, ie, the ability to share the emotional experience of another person, contributes importantly to appraisal processes and emotional reactions especially in stressful situations. Obviously, the degree of emotional empathy that a person is feeling (or not feeling) in a given situation modulates the appraisal of that situation and response strategies (Ter Horst et al, 2012; de Kloet, 2013; Kruk et al, 2013). However, at present, it remains unclear why MR stimulation improves emotional but not cognitive empathy and further studies need to replicate and further explore these findings (Zhou et al, 2011).

Our study has potentially important clinical implications. Intranasal oxytocin, a peptide that exerted positive effects on empathy (Meyer-Lindenberg et al, 2011), has already been used in pilot studies to examine whether there are synergistic effects of oxytocin and psychotherapy in depressed patients (Macdonald et al, 2013). Similarly, stimulation of MR could be potentially used to enhance emotional empathy and thus, therapeutic alliance and learning effects in psychotherapeutic settings. Preclinical animal data are consistent with this idea, because female mice with forebrain-specific deletion of the MR gene were unable to show extinction of contextual fear, and could not discriminate between cue and context fear unlike control mice (Ter Horst et al, 2012). In contrast, forebrain MR overexpression enhanced memory and reduced anxiety (Lai et al, 2007; Rozeboom et al, 2007). Therefore, a combination of MR stimulation with psychotherapeutic interventions might provide new avenues for a better treatment of BPD that is characterized by disturbed emotion regulation and emotional social interaction pathology.

We did not find significant differences between BPD patients and healthy women with regard to cognitive and 
emotional empathy across treatment modalities in our study as suggested by some, but not all, previous studies (Dziobek et al, 2011; Schilling et al, 2012). Of note, some authors even discussed increased cognitive and emotional empathy in BPD (Roepke et al, 2012; Dinsdale and Crespi, 2013). In our study we found, on a descriptive level, diminished emotional empathy in BPD with an effect size of Cohen's $d=0.2$ in the placebo condition, which corresponds to a small effect. It might be also the case that the used tasks that have been developed for autism spectrum disorders are not specific enough to detect BPD specific alterations in empathy, as also suggested for other tasks in the context of social cognition, eg, the 'Reading the Mind in the Eyes Test' (Schilling et al, 2012). Of note, using the MASC, has been shown that comorbid PTSD strongly influences cognitive empathy in a sample of BPD patients (Preissler et al, 2010). Thus, the relatively low prevalence of PTSD in the current study might be one reason for the unimpaired performance of the BPD patients in cognitive empathy. Furthermore, cognitive and emotional empathy in a given situation might depend on the level of arousal in that situation with decreasing empathy capacities in the face of increasing arousal (Dziobek et al, 2011; Ripoll et al, 2013). In our study, no stress paradigm was used and future studies should further examine the association between arousal and empathy in BPD.

There are some limitations of the study to be mentioned. First, the sample was too small to perform subgroup analyses, eg, with regard to comorbid mental disorders as major depressive disorder or PTSD. These disorders are known to influence cognitive empathy and HPA axis (Wingenfeld et al, 2010; Roepke et al, 2012; Wingenfeld et al, 2013). Furthermore, only women were included in this study and, therefore, no conclusions can be drawn with regard to men. Of note, first animal data indicate that MR mediates differences in emotional and cognitive behaviors between female and male mice (Ter Horst et al, 2012). Thus, our results need replication in a larger sample addressing comorbidities and potential sex effects. Menstrual cycle phase was not controlled for. However, intake of oral contraceptive did not influence the results. Furthermore, we did not ask the participant about side effects or if they had any hypotheses whether they received active treatment or not. Finally, although fludrocortisone has an about 15-fold higher affinity to MR compared with GR (Agarwal et al, 1977), we cannot completely rule out some GR effects of fludrocortisone. Dose-response studies are needed in this regard to disentangle MR from GR effects. Furthermore, we did not include a measure of current mood states during the examination. Thus, we cannot rule out that our results are secondary to an overall induced emotionality. In addition, further studies should include other measures of empathy and compare BPD patients and healthy participants with high emotional empathy scores with those with lower emotional empathy with respect to their response to MR stimulation. Future studies should examine whether fludrocortisone affects mood state and arousal of the participants and whether this is related to the degree of empathy.

In summary, this is the first study demonstrating enhanced emotional empathy after MR stimulation in BPD patients and healthy women. Clearly, further research on MR function and potential therapeutic effects of MR stimulation on emotional and cognitive processes in stress-related disorders such as BPD is warranted.

\section{FUNDING AND DISCLOSURE}

The authors declare no conflict of interest.

\section{ACKNOWLEDGEMENTS}

The study was supported by grant of the Deutsche Forschungsgemeinschaft (OT 209/7-1) awarded to CO. Furthermore, this work was supported by the DFG (EXC 257 NeuroCure).

\section{REFERENCES}

Ackenheil M, Stotz G, Dietz-Bauer R, Vossen A (1999). Mini International Neuropsychiatric Interview. German Version 5.0.0, DSM-IV. München, Psychiatrische Universitätsklinik München.

Agarwal MK, Coupry F, Philippe M (1977). Physiological activity and receptor binding of 9 alpha fluorohydrocortisone. Biochem Biophys Res Commun 78: 747-753.

Blair RJ (2008). Responding to the emotions of others: dissociating forms of empathy through the study of typical and psychiatric populations. Conscious Cogn 14: 698-718.

Brinks V, van der Mark MH, de Kloet ER, Oitzl MS (2007). Differential MR/GR activation in mice results in emotional states beneficial or impairing for cognition. Neural Plast 2007: 90163.

Cornelisse S, Joels M, Smeets T (2011). A randomized trial on mineralocorticoid receptor blockade in men: effects on stress responses, selective attention, and memory. Neuropsychopharmacology 36: 2720-2728.

de Kloet ER (2010). From vasotocin to stress and cognition. Eur J Pharmacol 626: 18-26.

de Kloet ER (2013). Functional profile of the binary brain corticosteroid receptor system: mediating, multitasking, coordinating, integrating. Eur J Pharmacol 719: 53-62.

de Kloet ER, Joels M, Holsboer F (2005). Stress and the brain: from adaptation to disease. Nat Rev Neurosci 6: 463-475.

Dinsdale N, Crespi BJ (2013). The borderline empathy paradox: evidence and conceptual models for empathic enhancements in borderline personality disorder. J Pers Disord 27: 172-195.

Dziobek I, Fleck S, Kalbe E, Rogers K, Hassenstab J, Brand M et al (2006). Introducing MASC: a movie for the assessment of social cognition. J Autism Dev Disord 36: 623-636.

Dziobek I, Preissler S, Grozdanovic Z, Heuser I, Heekeren HR, Roepke S (2011). Neuronal correlates of altered empathy and social cognition in borderline personality disorder. Neuroimage 57: 539-548.

Dziobek I, Rogers K, Fleck S, Bahnemann M, Heekeren HR, Wolf OT et al (2008). Dissociation of cognitive and emotional empathy in adults with Asperger syndrome using the Multifaceted Empathy Test (MET). J Autism Dev Disord 38: 464-473.

First MB, Spitzer RL, Gibbon M, Williams JBW et al (1997). Structured clinical interview for DSM-IV (R Axis I disorders (SCID-I). Clinician Version.

Groeneweg FL, Karst H, de Kloet ER, Joëls M (2012). Mineralocorticoid and glucocorticoid receptors at the neuronal membrane, regulators of nongenomic corticosteroid signalling. Mol Cell Endocrinol 350: 299-309.

Harris AP, Holmes MC, de Kloet ER, Chapman KE, Seckl JR (2012). Mineralocorticoid and glucocorticoid receptor balance in control of HPA axis and behaviour. Psychoneuroendocrinology 38: 648-658. 
Hurlemann R, Hawellek B, Maier W, Dolan RJ (2007). Enhanced emotion-induced amnesia in borderline personality disorder. Psychol Med 37: 971-981.

Joels M, Fernandez G, Roozendaal B (2011). Stress and emotional memory: a matter of timing. Trends Cogn Sci 15: 280-288.

Joels M, Karst H, DeRijk R, de Kloet ER (2008). The coming out of the brain mineralocorticoid receptor. Trends Neurosci 31: 1-7.

Kruk MR, Haller J, Meelis W, de Kloet ER (2013). Mineralocorticoid receptor blockade during a rat's first violent encounter inhibits its subsequent propensity for violence. Behav Neurosci 127: 505-514.

Lai M, Horsburgh K, Bae SE, Carter RN, Stenvers DJ, Fowler JH et al (2007). Forebrain mineralocorticoid receptor overexpression enhances memory, reduces anxiety and attenuates neuronal loss in cerebral ischaemia. Eur J Neurosci 25: 1832-1842.

Lupien SJ, Lepage M (2001). Stress, memory, and the hippocampus: can't live with it, can't live without it. Behav Brain Res 127: $137-158$.

Macdonald K, Macdonald TM, Brüne M, Lamb K, Wilson MP, Golshan S et al (2013). Oxytocin and psychotherapy: a pilot study of its physiological, behavioral and subjective effects in males with depression. Psychoneuroendocrinology 38: 2831-2843.

Meyer-Lindenberg A, Domes G, Kirsch P, Heinrichs M (2011). Oxytocin and vasopressin in the human brain: social neuropeptides for translational medicine. Nat Rev Neurosci 12: 524-538.

Otte C, Moritz S, Yassouridis A, Koop M, Madrischewski AM, Wiedemann $\mathrm{K}$ et al (2007). Blockade of the mineralocorticoid receptor in healthy men: effects on experimentally induced panic symptoms, stress hormones, and cognition. Neuropsychopharmacology 32: 232-238.

Preissler S, Dziobek I, Ritter K, Heekeren HR, Roepke S (2010). Social cognition in borderline personality disorder: evidence for disturbed recognition of the emotions, thoughts, and intentions of others. Front Behav Neurosci 4: 182.

Rimmele U, Besedovsky L, Lange T, Born J (2013). Blocking mineralocorticoid receptors impairs, blocking glucocorticoid receptors enhances memory retrieval in humans. Neuropsychopharmacology 38: 884-894.

Ripoll LH, Snyder R, Steele H, Siever LJ (2013). The neurobiology of empathy in borderline personality disorder. Curr Psychiatry Rep 15: 344 .
Ritter K, Dziobek I, Preissler S, Rüter A, Vater A, Fydrich T et al (2011). Lack of empathy in patients with narcissistic personality disorder. Psychiatry Res 187: 241-247.

Roepke S, Vater A, Preißler S, Heekeren HR, Dziobek I (2012). Social cognition in borderline personality disorder. Front Neurosci 6: 195.

Roozendaal B, Hernandez A, Cabrera SM, Hagewoud R, Malvaez M, Stefanko DP et al (2010). Membrane-associated glucocorticoid activity is necessary for modulation of long-term memory via chromatin modification. J Neurosci 30: 5037-5046.

Rozeboom AM, Akil H, Seasholtz AF (2007). Mineralocorticoid receptor overexpression in forebrain decreases anxiety-like behavior and alters the stress response in mice. Proc Natl Acad Sci USA 104: 4688-4693.

Schilling L, Wingenfeld K, Löwe B, Moritz S, Terfehr K, Köther U et al (2012). Normal mind-reading capacity but higher response confidence in borderline personality disorder patients. Psychiatry Clin Neurosci 66: 322-327.

Sheehan DV, Lecrubier Y, Sheehan KH, Amorim P, Janavs J, Weiller E et al (1998). The Mini-International Neuropsychiatric Interview (M.I.N.I.): the development and validation of a structured diagnostic psychiatric interview for DSM-IV and ICD-10. J Clin Psychiatry 59(Suppl 20): 22-33 quiz 34-57.

Smeets T, Dziobek I, Wolf OT (2009). Social cognition under stress: differential effects of stress-induced cortisol elevations in healthy young men and women. Horm Behav 55: 507-513.

Ter Horst JP, Carobrez AP, van der Mark MH, de Kloet ER, Oitzl MS (2012). Sex differences in fear memory and extinction of mice with forebrain-specific disruption of the mineralocorticoid receptor. Eur J Neurosci 36: 3096-3102.

Wingenfeld K, Driessen M, Terfehr K, Schlosser N, Fernando SC, Otte $C$ et al (2013). Effects of cortisol on memory in women with borderline personality disorder: role of co-morbid post-traumatic stress disorder and major depression. Psychol Med 43: 495-505.

Wingenfeld K, Spitzer C, Rullkötter N, Löwe B (2010). Borderline personality disorder: hypothalamus pituitary adrenal axis and findings from neuroimaging studies. Psychoneuroendocrinology 35: 154-170.

Zaki J, Ochsner K (2012). The neuroscience of empathy: progress, pitfalls and promise. Nat Neurosci 15: 675-680.

Zhou M, Kindt M, Joëls M, Krugers HJ (2011). Blocking mineralocorticoid receptors prior to retrieval reduces contextual fear memory in mice. PLoS One 6: e26220. 